\title{
Magnon Accumulation by Clocked Laser Excitation as Source of Long-Range Spin Waves in Transparent Magnetic Films
}

\author{
M. Jäckl, ${ }^{1}$ V. I. Belotelov, ${ }^{2,3}$ I. A. Akimov, ${ }^{1,4}$ I. V. Savochkin, ${ }^{2}$ D. R. Yakovlev, ${ }^{1,4}$ A. K. Zvezdin, ${ }^{3,5,6}$ and M. Bayer ${ }^{1,4}$ \\ ${ }^{1}$ Experimentelle Physik 2, Technische Universität Dortmund, D-44221 Dortmund, Germany \\ ${ }^{2}$ Lomonosov Moscow State University, 119991 Moscow, Russia \\ ${ }^{3}$ Russian Quantum Center, Skolkovo, 143025 Moscow, Russia \\ ${ }^{4}$ Ioffe Institute, Russian Academy of Sciences, 194021 St. Petersburg, Russia \\ ${ }^{5}$ Moscow Institute of Physics and Technology, Moscow Region 141700, Russia \\ ${ }^{6}$ Prokhorov General Physics Institute, Russian Academy of Sciences, 119991 Moscow, Russia
}

(Received 17 January 2017; revised manuscript received 23 February 2017; published 19 April 2017)

\begin{abstract}
Optical tools are promising for spin-wave generation because of the possibilities of ultrafast manipulation and local excitation. However, a single laser pulse can inject spin waves (SWs) only with a broad frequency spectrum, resulting in short propagation distances and low wave amplitudes. Here, we excite a magnetic garnet film by a train of fs-laser pulses with a 1-GHz repetition rate so that the pulse separation is shorter than the decay time of magnetic modes, which allows us to achieve a collective impact on the magnetization and establish a quasistationary source of spin waves, namely, a coherent accumulation of magnons ("magnon cloud"). This approach has several appealing features: (i) The magnon source is tunable, (ii) the SW amplitude can be significantly enhanced, (iii) the SW spectrum is quite narrow, providing long-distance propagation, (iv) the periodic pumping results in an almost constant-in-time SW amplitude for the distances larger than $20 \mu \mathrm{m}$ away from the source, and (v) the SW emission shows pronounced directionality. These results expand the capabilities of ultrafast coherent optical control of magnetization and pave the way for applications in data processing, including the quantum regime. The quasistationary magnon accumulation might also be of interest for applications in magnon Bose-Einstein condensates.
\end{abstract}

DOI: 10.1103/PhysRevX.7.021009

Subject Areas: Magnetism, Optics

\section{INTRODUCTION}

Recent research on spin waves (SWs) is increasingly driven by their unique linear and nonlinear properties as well as anticipated applications in telecommunication, image processing, and even quantum computation [1-5]. SWs are launched if, in a magnetically ordered material, the magnetization is pushed out of equilibrium. Usually, this is achieved by microwaves generated by an antenna in close vicinity to the sample [6]. However, particular applications require a strong locality of the excitation and a specific distribution of spins in time and space, created on time scales much shorter than any decay time. For example, quantum information processing necessitates addressing a qubit by a magnetic field with a submicron gradient [5]. This challenge might be solved if the magnetic system is disturbed by ultrashort laser pulses that can be focused microscopically [7-24]. Then, the instantaneous impact of the laser pulse on the magnetization occurs only within the

Published by the American Physical Society under the terms of the Creative Commons Attribution 4.0 International license. Further distribution of this work must maintain attribution to the author(s) and the published article's title, journal citation, and DOI. illumination spot with potentially subwavelength resolution if plasmonic nanogeometries are used [25,26].

Among the various mechanisms for optical pumping of magnetization in ferromagnets, the inverse Faraday effect is of particular importance [8,20-23]. Here, circularly polarized light affects the medium magnetization, as an effective magnetic field $\mathbf{H}_{F} \sim\left[\mathbf{E} \times \mathbf{E}^{*}\right]$ would act, where $\mathbf{E}$ is the electric field of the light wave [27]. As a result, $\mathbf{H}_{F}$ is directed along the light wave vector. In magnetically ordered media, it originates from stimulated Raman scattering on magnons. The inverse Faraday effect was observed in pump-probe experiments [20-23], where the magnetization dynamics is triggered by a pump and subsequently monitored by a delayed probe pulse of low intensity. The authors of Refs. [8-10] managed to demonstrate magnetostatic SWs in iron garnets in that way.

Almost all pump-probe studies so far were conducted in the single-pump-pulse regime, where the magnetization oscillations decay before the subsequent pulse arrives. Double-pump coherent magnetization control was demonstrated in Refs. [20] and [28]. Recently, the magnetization precession was excited by a sequence of picosecond acoustic pulses [29]. However, so far there have been no studies of the impact of a virtually infinite sequence of pump pulses exciting the sample at such a high rate that 
upon pulse arrival the magnetization still shows the coherent dynamics induced by the preceding pulse.

Here, we excite transparent magnetic films with a train of fs-laser pulses periodically hitting the sample with a period of $1 \mathrm{~ns}$. This pulse separation is comparable to or even shorter than the decay time of the magnetization modes. Being periodic in time, the excitation generates a field $\mathbf{H}_{F}(t)$ in the illuminated area, which represents a quasicontinuous source of SWs showing several appealing features, namely, directionality of the SWs as well as enhanced amplitudes for specific SW frequencies, providing frequency selectivity. This magnetization amplification facilitates observation of usually inaccessible SW modes.

\section{MATERIALS AND METHODS}

The experiments here are conducted on monocrystalline ferrimagnet films of bismuth-substituted iron garnet: a 5- $\mu \mathrm{m}$-thick film with chemical composition $\left(\mathrm{Bi}_{0.9} \mathrm{Lu}_{1.4} \mathrm{Tm}_{0.4} \mathrm{Y}_{0.2} \mathrm{Sm}_{0.1}\right)\left(\mathrm{Fe}_{4.7} \mathrm{Ga}_{0.3}\right) \mathrm{O}_{12}$ (sample 1, Figs. 1 and 4 ) and a $4-\mu$ m-thick film of composition
$\left(\mathrm{Bi}_{0.8} \mathrm{Lu}_{2.2}\right) \mathrm{Fe}_{5} \mathrm{O}_{12}$ (sample 2, Figs. 2 and 3). Both films were grown by liquid phase epitaxy on gadolinium gallium garnet (GGG) with crystallographic orientation (111).

The magnetization precession is excited and detected using a pump-probe technique based on asynchronous optical sampling [31,32]. Two independent Ti:Sapphire laser oscillators (Gigajet TWIN 20c/20c) for the circularly polarized pump and the linearly polarized probe beams emit 50-fs pulses with a center wavelength of $\lambda \approx 800 \mathrm{~nm}$ at a rate of about $f=1 \mathrm{GHz}$ [Fig. 1(a)]. The repetition frequencies of the oscillators are synchronized to each other with a small offset of $\Delta f=2 \mathrm{kHz}$ using TL-1000 ASOPS unit. As a result, the relative time delay between pump and probe pulses is repetitively ramped from zero to $1 \mathrm{~ns}$ within a scan time of $5 \mu \mathrm{s}$. In this case, the ultrafast signal is linearly stretched in time by a factor of about $f / \Delta f$, which makes it accessible to fast data acquisition electronics. The pump and probe beams are focused onto the sample using a single reflective microscope objective with a magnification factor of 15 , comprising four sectors through which the
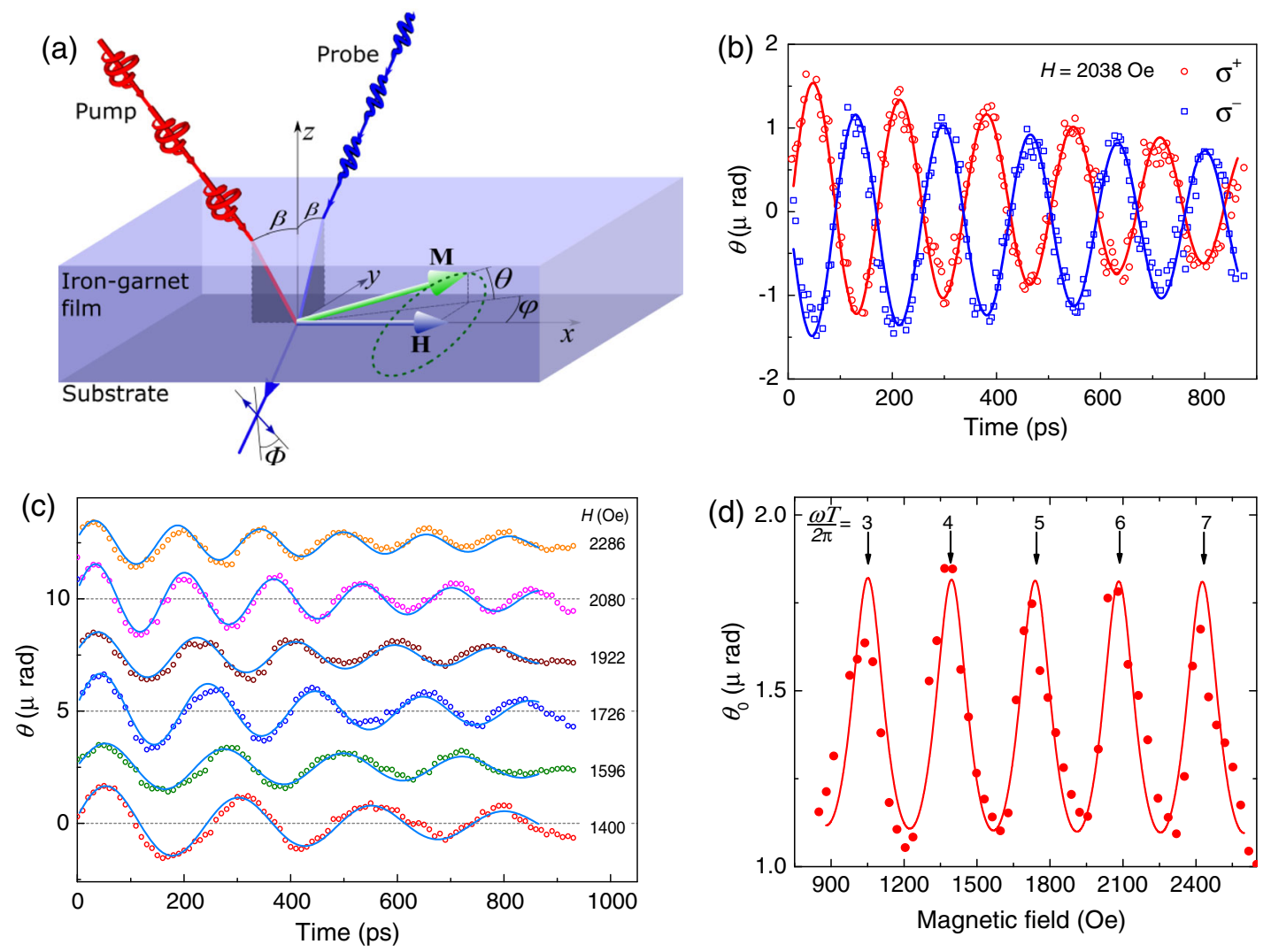

FIG. 1. Excitation of magnetization precession in an iron-garnet film by a train of fs optical pulses. (a) Experimental scheme. The periodic laser pulses are incident on the magnetic film. The probe beam is detected from the substrate side of the sample. (b) Comparison of magnetization oscillations excited by $\sigma^{+}$and $\sigma^{-}$circularly polarized pumps (red circles and blue squares, respectively). Solid curves are fits with a decaying sine function. The fit parameters are given in Table SI (see Ref. [30]), $H=2038$ Oe. (c) Magnetization oscillations at different magnetic fields (open symbols) corresponding to maximal and minimal amplitudes. Fits to the data by decaying sines are given by the thin blue curves. The incident light is $\sigma^{+}$polarized. Fit parameters are given in Table SII (see Ref. [30]). (d) Measured precession amplitude vs magnetic field strength (circles). The solid curve is calculated after Eq. (3) using the parameters $h=6.2 \mathrm{Oe}, \alpha=0.02(\tau=0.77 \mathrm{~ns}$ at $H=1500 \mathrm{Oe})$ to fit the experimental data. Experimental results are presented for sample 1 . 

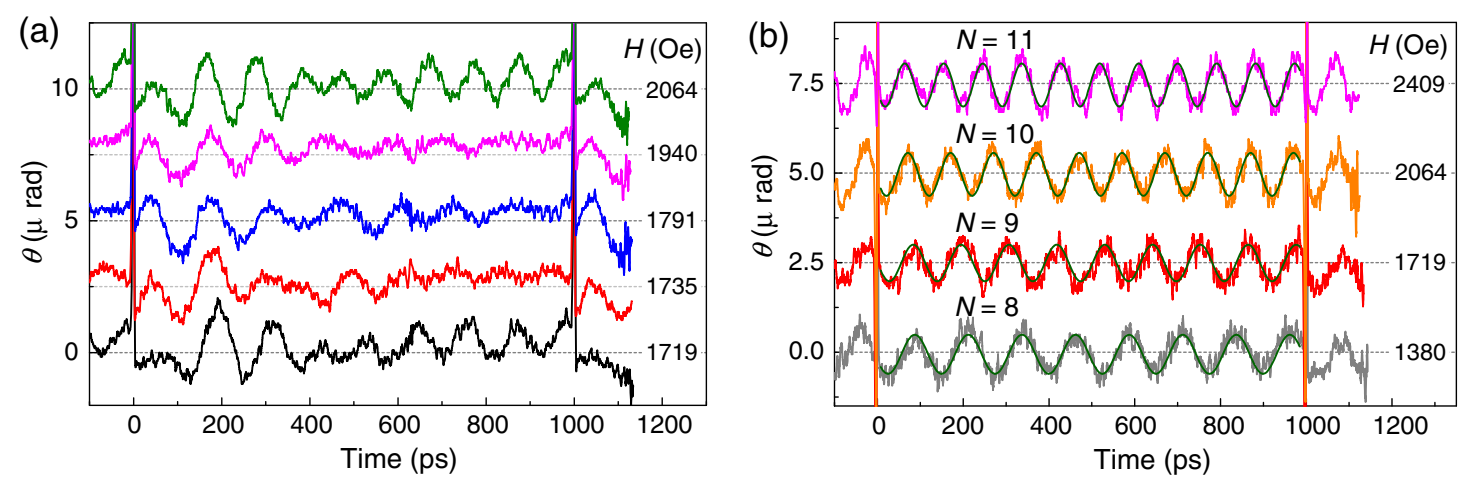

FIG. 2. Excitation of magnetization oscillation showing two precession modes in an iron-garnet film by a laser pulse train. (a) Magnetization oscillations at different magnetic fields. (b) Consecutive resonances of the slowly decaying precession mode B for different magnetic fields. The green thin lines are fits with decaying sine functions. The fit parameters are given in Table SIII (see Ref. [30]). The experimental data are presented for sample 2.

light can enter and exit. The second microscope objective is used to collect and collimate the probe beam in transmission geometry. Pump and probe beams are incident at an angle $\beta=17^{\circ}$ in planes orthogonal to each other [the $X Z$ and $Y Z$ planes in Fig. 1(a)]. The pump pulses with energy of around $50 \mathrm{pJ}$ are focused into a spot size of about $10 \mu \mathrm{m}$ diameter, while the probe pulses with energy of about $3 \mathrm{pJ}$ are focused into a spot of about $7 \mu \mathrm{m}$ diameter.

Variations of the out-of-plane magnetization component, i.e., the component along the $z$ axis, are detected by the Faraday rotation angle $\Phi$ of the probe beam with variable
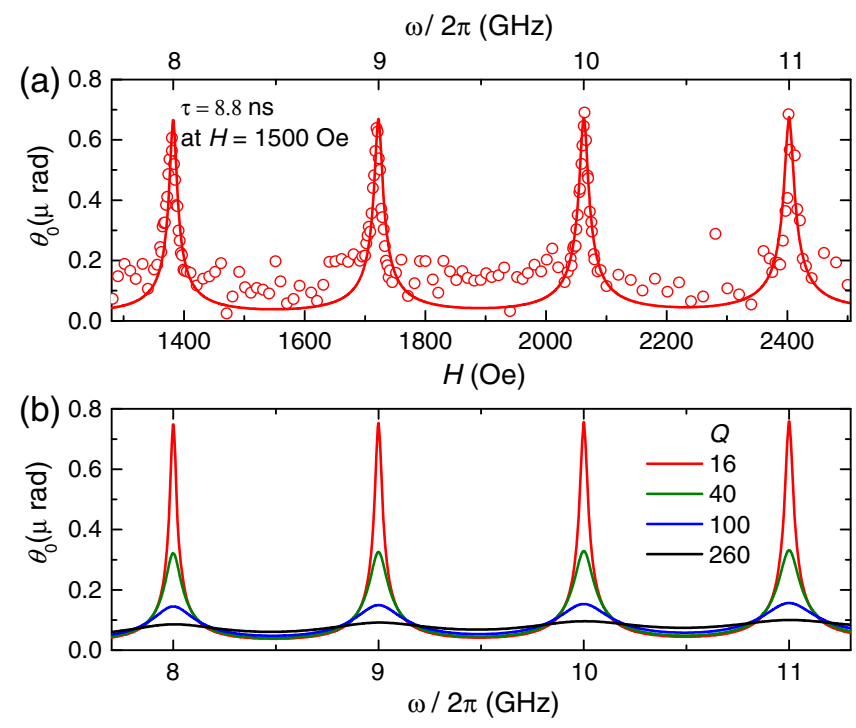

FIG. 3. Excitation of magnetization oscillation mode of high quality factor (mode B). (a) Measured precession amplitude in sample 2 vs magnetic field (open circles). The solid curve is calculated by solving Eq. (S11) (see Ref. [30]) with $h=0.8$ Oe, and $\alpha=0.005(\tau=8.8 \mathrm{~ns}$ at $H=1500 \mathrm{Oe})$ to fit the experimental data. (b) Calculated precession amplitude vs $\omega$ for modes of different quality factors [and therefore different values of $\tau$ : $0.6 \mathrm{~ns}$ (black line), $1.5 \mathrm{~ns}$ (blue line), $4 \mathrm{~ns}$ (green line), and $10 \mathrm{~ns}$ (red line) at $H=1500$ Oe]. Here, $h=0.8$ Oe. time delay relative to the pump. The angle $\Phi$ is measured using a polarization bridge that comprises a Wollaston prism and a 10-MHz balanced photodetector. The amplified differential signal is sent to a high-speed multichannel digitizer triggered by the asynchronous-optical-sampling system at a frequency of $2 \mathrm{kHz}$. The resulting Faraday rotation angle is given by $\theta(t)=d U(t) / 4 U_{\mathrm{dc}}$, where $d U(t)$ is the time-resolved differential signal and $U_{\mathrm{dc}}$ is the average intensity measured with one of the photodiodes (see Sec. 1 in Ref. [30]). The magnetization precession angle $\theta$ can be deduced from $\Phi$ [Eq. (S3), Ref. [30]]. The external magnetic field up to $2500 \mathrm{Oe}$ is applied in plane, along the $x$ axis, of the sample using an electromagnet. All measurements are performed at room temperature.

For numerical calculation of spin-wave amplitude and dispersion [see Figs. 4(b) and 4(c)], we used the following magnetic parameters of the $5-\mu \mathrm{m}$-thick film: gyromagnetic ratio $\gamma=1.76 \times 10^{7} \mathrm{rad} \mathrm{Oe}^{-1} \mathrm{~s}^{-1}$, saturation magnetization $4 \pi M_{s}=1000 \mathrm{Oe}$, uniaxial anisotropy constant $K_{u}=3.0 \times 10^{4} \mathrm{erg} \mathrm{cm}^{-3}$, and cubic anisotropy constant $K_{1}=-1.2 \times 10^{3} \mathrm{erg} \mathrm{cm}^{-3}$.

\section{RESULTS AND DISCUSSION}

\section{A. Collective photonic impact on magnetization}

The train of circularly polarized pump pulses excites a periodic magnetization dynamics [Fig. 1(b)]. Different mechanisms might be responsible for the optical excitation of the magnetization oscillations in magnetic dielectrics, including the inverse magneto-optical effects related to the impulsive stimulated Raman scattering (ISRS), photoinduced magnetocrystalline anisotropy, and thermal effects $[33,34]$. The pump wavelength $(\lambda=800 \mathrm{~nm})$ is in the transparency wavelength range of the samples, with an absorption coefficient of about $90 \mathrm{~cm}^{-1}$ that allows us to neglect thermal effects in our case. The oscillation amplitude is directly proportional to the pump fluence (Fig. 1S, Ref. [30]). Moreover, switching the pump helicity from $\sigma^{+}$ 

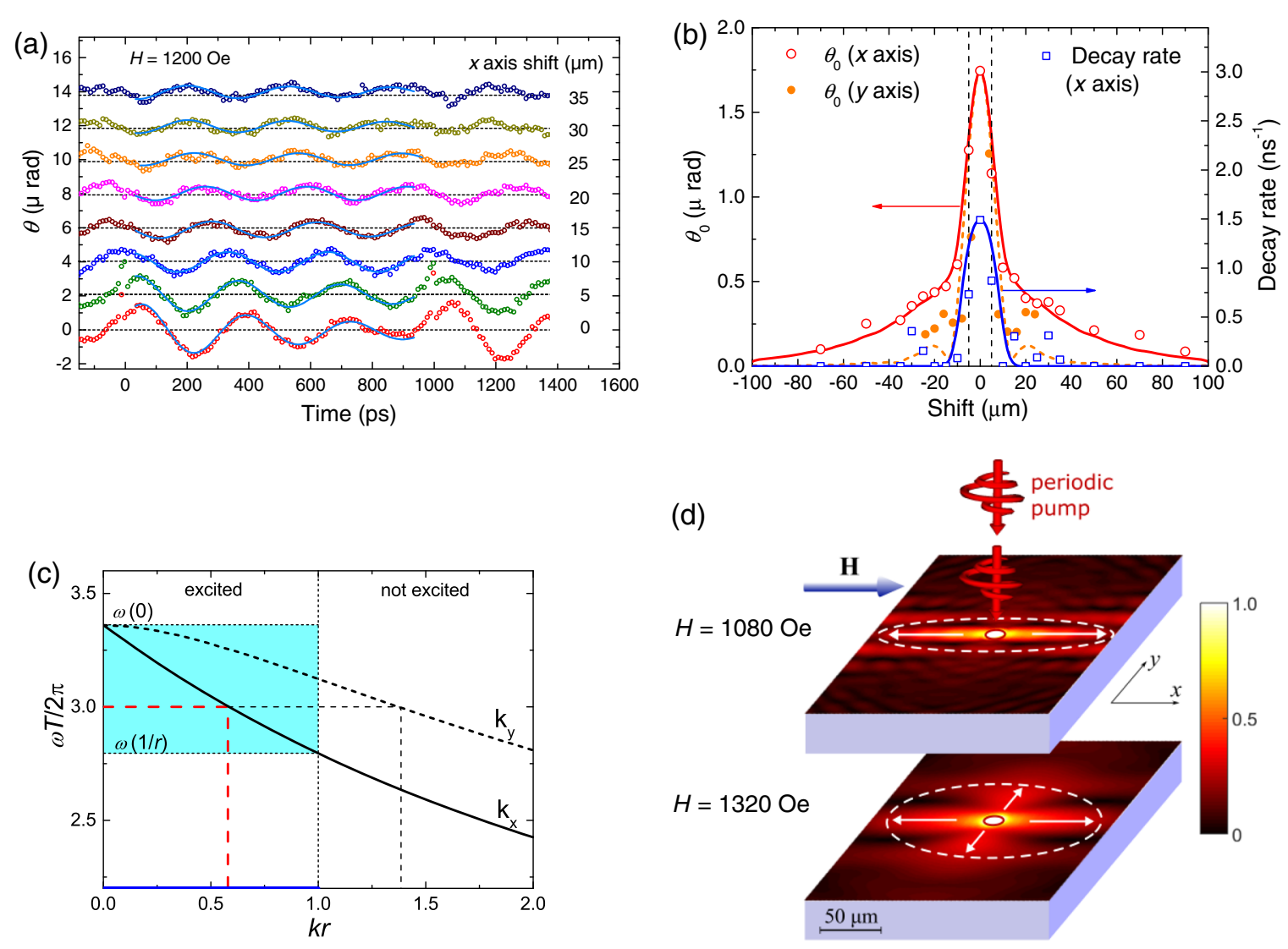

FIG. 4. Generation of spin waves by a cloud of periodically pumped, coherently oscillating spins. (a) Magnetization oscillations observed in sample 1 in the probe beam shifted by 5-35 $\mu \mathrm{m}$ relative to the pump beam along the external magnetic field. Solid curves are fits with a decaying sine function. The fit parameters are given in Table SII (see Ref. [30]). Here, $H=1060$ Oe. (b) Oscillation amplitude (red and orange) and decay rate (blue) at different distances between the pump and probe for the probe shifted along the $x$ axis (open symbols and solid lines) and the $y$ axis (filled symbols and dashed lines). The experimental data are shown by the symbols, while the calculation results are given by the lines. (c) Calculated dispersion of the SWs propagating along the $x$ and $y$ directions. The blue region indicates the range of $k$ generated by a single laser pulse with spot radius $r$. The dispersion was calculated using the theoretical model in Ref. [35]. (d) Calculated distribution of $m_{z}$ in SWs launched at $H=1060$ Oe $(\omega T / 2 \pi=3)$ and $H=1320$ Oe $(\omega T / 2 \pi=3.75)$. In the former case, SWs are generated in a narrow region along $\mathrm{H}$ (phenomenon of SW directionality), while in the latter one, SWs are generated in the perpendicular direction as well.

to $\sigma^{-}$changes the precession phase by $\pi$ [Fig. 1(b)]. On the other hand, the birefringence of the iron garnet films is negligibly small. Therefore, the ellipticity of the refracted waves is also negligible, and we assume that, in our case, the inverse Faraday effect is responsible for the oscillation excitation.

Sweeping the external magnetic field modifies the amplitude $\theta_{0}$, phase $\zeta$, and frequency $\omega$ of the oscillations. In the time interval of $T=1 \mathrm{~ns}$ between two consecutive laser pulses, the oscillations can be approximately described by a decaying sine function: $\theta=\theta_{0} e^{-t / \tau} \sin (\omega t+\zeta)$ [Fig. 1(c)], where $\tau$ is the decay time. Small deviations of the measured data from the fit curves are caused by excitation of SWs. Strikingly, the precession amplitude shows resonances for particular magnetic fields, at which the oscillation frequency is a multiple of the laser repetition rate, corresponding to the following relation: $\omega T / 2 \pi=N$, where $N$ is an integer [Fig. 1(d)]. Accordingly, for $N=4,5,6$, and 7, the resonances appear at $H=1400,1726,2080$, and 2427 Oe, respectively. At such a resonance, $\theta_{0}$ exceeds its minimal value by $60 \%$ and $\zeta$ vanishes [see blue curve at $H=1400$ Oe in Fig. 1(c)], so there is no phase shift, which is the result of spin synchronization: The oscillations at each excitation cycle are synchronized with each other.

For some samples, the magnetization precession does not occur on a single frequency [see Fig. 2(a)]. For all magnetic fields shown there, the signal contains a fastdecaying component, termed mode $\mathrm{A}$ in the following, with a decay time of about $0.7 \mathrm{~ns}$, while for some resonant magnetic fields (e.g., for $H=1719$ Oe or 2064 Oe), an additional, slowly decaying component, mode $\mathrm{B}$, can be clearly identified at probe-pump delays exceeding $0.7 \mathrm{~ns}$. Mode B disappears completely for even slight detuning from the resonant fields (compare the oscillations at $H=$ 1719 Oe and 1735 Oe). By subtracting the off-resonant signals from the resonant ones, one can isolate mode B, 
which almost does not decay in the 1-ns time interval. Four consecutive mode-B resonances corresponding to $N=8$, 9, 10, and 11 are shown in Fig. 2(b).

The amplitude of mode A behaves quite similarly to the mode discussed above in Fig. 1. However, the mode-B amplitude changes with magnetic field much more prominently [Fig. 3(a)]. In resonance where its frequency is a multiple of the excitation rate, the mode-B amplitude is 5 times higher than the noise level and comparable to the mode-A amplitude. Remarkably, in the single-pulse excitation regime, i.e., when the sample is excited at a much lower repetition rate $(1 / T=80 \mathrm{MHz})$, the amplitude of mode B is much smaller than that of mode A and, therefore, cannot be resolved.

\section{B. Analysis of the observed phenomena}

A quantitative understanding of the observations can be obtained from the dynamical equation for the magnetization. The excitation by optical pulses generally leads to a spatial and temporal pattern of the magnetization $\mathbf{M}(\mathbf{r}, t)$. The magnetization dynamics of these modes is described by the Landau-Lifshitz-Gilbert equation:

$$
\frac{d \mathbf{M}_{q}}{d t}=-\gamma \mathbf{M} \times \mathbf{H}_{\mathrm{eff}}+\frac{\alpha}{M} \mathbf{M} \times \frac{d \mathbf{M}}{d t},
$$

where $\gamma$ is the gyromagnetic ratio and $\alpha$ is the Gilbert damping constant. The effective magnetic field $\mathbf{H}_{\text {eff }}$ in this equation is determined by the variational derivative of the sample free energy $W$ with respect to the magnetization: $\mathbf{H}_{\text {eff }}=-\delta W / \delta \mathbf{M}$. The free energy is the sum of several contributions: $W=W_{Z}+W_{d}+W_{a}+W_{F}$, where $W_{Z}$ is the Zeeman energy in the external magnetic field $\mathbf{H}$, and $W_{d}$ and $W_{a}$ are the demagnetization and magnetic anisotropy energies, respectively. The last term, $W_{F}$, takes into account the inverse Faraday effect: $W_{F}=-\mathbf{M} \cdot \mathbf{H}_{F}$. When a laser pulse propagates through the magnetic film at an angle $\beta$ relative to its normal and $\mathbf{H}$ has components both in the sample and the light incidence planes, Eq. (1) leads to (see Ref. [30])

$$
\begin{aligned}
& \frac{\partial^{2} \theta}{\partial t^{2}}+\alpha\left(\omega_{H}+\omega_{e}\right) \frac{\partial \theta}{\partial t}+\left(\omega_{H}+\omega_{F \perp}(t)\right)\left(\omega_{e}+\omega_{F \perp}(t)\right) \theta \\
& \quad=\omega_{H} \omega_{F \|}(t)
\end{aligned}
$$

where $\omega_{H}=\gamma H, \omega_{e}=\gamma\left(H+4 \pi M-2 K_{u} / M\right), \omega_{F \|}(t)=$ $\gamma H_{F}(t) \cos \beta$, and $\omega_{F \perp}(t)=\gamma H_{F}(t) \sin \beta$.

If magnetic losses are neglected and $\beta \rightarrow \pi / 2$, Eq. (2) becomes isomorphic to the Schrödinger equation with a periodic potential $V(t)=-\gamma H_{F}(t)\left(\omega_{0}+\omega_{e}\right)$, and the problem is reduced to the Kronig-Penney model with eigenfrequencies $\omega_{0} \omega_{e}$. For relatively large $H_{F}$, band gaps in the oscillation spectrum appear, so appealing effects such as the parametric generation of magnetization oscillations become possible.
In our experimental conditions, the laser pulses propagate through the film almost along its normal so that $\beta \ll 1$. In this case, the right-hand side of Eq. (2) becomes prominent, and the inverse Faraday effect acts on the magnetization as an external periodic force. Since the optical pulse duration $\Delta t \ll T, \omega_{H}^{-1}$, the following representation of this force can be used: $\omega_{F \|}(t)=$ $\gamma \Delta t \sum_{m=0}^{+\infty} h_{m} \delta(t-m T)$, where $h_{m}$ is the amplitude of the $m$ th pulse in $H_{F}$.

The problem can be solved using the Green function formalism: $\quad \theta(t)=\frac{1}{\omega} \sum_{m=0}^{+\infty} h_{m} G(t+m T)$, where the Green function $G(t)=\omega^{-1} \sin \omega t e^{-t / \tau}$ for $t \geq 0$, $G(t)=0$ for $t<0$, and $\tau=2 /\left(\alpha\left(\omega_{H}+\omega_{e}\right)\right)$. Here, $\omega=\omega_{0} \sqrt{1-\left(\omega_{0} \tau\right)^{-1}} \approx \omega_{0}$, where $\omega_{0}=\sqrt{\omega_{H} \omega_{e}}$. If the train of fs pulses is uniform, i.e., $h_{m}=h=$ const, then the oscillation regime becomes quasistationary and $\theta(t)$ is a periodic function of $T: \theta(t)=\theta_{0} \sin (\omega t+\zeta) e^{-t / \tau}$ within a single interval between two pump pulses, i.e., for $m T<t<(m+1) T, m \gg 1$. For relatively fast decay so that $e^{-T / \tau} \ll 1$, the amplitude and phase of the magnetization oscillation are given by (see Ref. [30])

$$
\left\{\begin{array}{l}
\theta_{0}=\frac{\omega_{H}}{\omega} \gamma h \Delta t\left(1+e^{-T / \tau} \cos \omega T\right) \\
\zeta=e^{-T / \tau} \sin \omega T
\end{array} .\right.
$$

Accordingly, the precession amplitude shows a resonance when the oscillations are synchronized with the laser pulses, i.e., at $\omega T=2 \pi N$. At these resonances, $\zeta=0$; i.e. the oscillations start with $\theta=0$ at the moment of subsequent pulse arrival, which is in good agreement with the experiment [Fig. 1(c)]. The ratio of the maximal and minimal amplitudes is given by

$$
\theta_{\max } / \theta_{\min }=\tanh (2 \tau / T)=\tanh \left(4 Q / \omega_{0} T\right)
$$

Therefore, the amplitude is increased most strongly for a magnetization mode of high quality factor $Q$ [Fig. 4(b)]. The enhancement factor relative to the amplitude $\theta_{s}$ of the oscillations excited by a single pump pulse is $\theta_{\max } / \theta_{s}=\left(1-e^{-T / \tau}\right)^{-1}$. Fitting the observed resonances at $H=1500$ Oe with the theoretical forms as shown in Figs. 1(d) and 4(a) gives $\tau=0.77 \mathrm{~ns}$ and $\tau=8.8 \mathrm{~ns}$ for mode A and mode B, respectively. These values correspond to enhancement factors of 1.4 and 9.3 for these two modes. This huge difference in the enhancement factors is a key point for the detection of mode B. In more detail, in the single-pulse regime, mode $\mathrm{A}$ is excited more efficiently than mode B. However, mode B has a much higher $Q$ factor, and therefore, in the multiple-pulse regime, it is enhanced 6.6 times more efficiently than mode A. As a result, mode $\mathrm{B}$ becomes visible as its amplitude becomes comparable to the amplitude of mode A. 


\section{Observation of tunable spin waves}

Let us now shift the probe beam with respect to the pump to trace the SWs. The oscillating signals observed for different pump-probe separations confirm propagation of the SWs in the direction along the field $\mathbf{H}$ [Fig. 4(a)]. SWs are detectable for spatial shifts up to $100 \mu \mathrm{m}$ [open red circles in Fig. 4(b)]. Notably, this distance is twice as large as for SWs excited in the single-pulse regime. On the contrary, in the orthogonal direction, the SWs are only observable within $10 \mu \mathrm{m}$ from the pump beam center [filled orange circles in Fig. 4(b)]. One more remarkable point is that the SW decay rate diminishes with increasing pumpprobe distance. For propagation distances larger than $10 \mu \mathrm{m}$, the rate undercuts $0.3 \mathrm{~ns}^{-1}$ [Fig. 4(b)], so the oscillations almost do not decay in time in between the pump pulses [Fig. 4(a)].

All these unique features of the periodic pumping of SWs are related again to the synchronization of oscillations and also involve the specific character of the SW dispersion. Since the Gilbert damping in iron garnets is relatively small, $\alpha \sim 10^{-3}$, which corresponds to $\tau \sim 100 \mathrm{~ns}$, the decay time is mainly determined by the spread of SW energy away from the excitation spot and the dephasing of SW modes of different frequencies due to the SW dispersion and group velocity. While the former is inevitable, the latter may be tailored as in our case by the periodic pumping because the repeated application of the pump pulses narrows the spectrum of generated SWs. Indeed, a single-pump pulse with spot radius $r$ excites SWs with wave numbers $k<1 / r$ corresponding to frequencies in the range $\omega(1 / r)<\omega<\omega(0)$ (for the backward magnetostatic SWs excited in our experiments) [Fig. 4(c)]. However, the train of pulses singles out frequencies that are multiples of the laser repetition rate $(1 / T)$ from this range (see red dashed line for $\omega=6 \pi / T$ ). Since the SW spectrum then becomes narrower, the dephasing decreases and the SWs propagate over longer distances.

As the SW spectrum is anisotropic with respect to $\mathbf{H}$ so that $\omega\left(k_{x}, 0\right) \neq \omega\left(0, k_{y}\right)$ [see Fig. 4(c)], the laser pulse train excites SWs along different directions with various efficiencies. For example, at $H=1200$ Oe, a multiple of the pulse repetition rate is within the frequency window of the excitable SWs along the $x$ axis [for $=0.6<1 \omega T / 2 \pi=3$, Fig. 2(c)], while there is no multiple in the SW frequency range along the $y$ axis [dashed curve in Fig. 2(c)]. As a result, SWs can be traced along the $x$ axis over distances of about $100 \mu \mathrm{m}$, but they can hardly be observed along the $y$ axis even for small distances from the pumped spot. Therefore, the periodic pumping introduces an additional potentiality as it excites SWs along specific directions which may be altered by changing the pulse repetition rate and the external magnetic field [Fig. 4(d)].

Finally, the vanishing time decay of the SWs at sufficiently large distances from the pump beam has to be explained. According to Fig. 4(a), in the optically excited area $(x=0)$, the observed signal is a superposition of the forced oscillations whose amplitude is constant in time and free oscillations that decay with $\tau=0.7 \mathrm{~ns}$. When shifting from the pumped area by more than $10 \mu \mathrm{m}$, the freeoscillation component fully decays, and only the forced oscillations are visible. Consequently, the magnons at such distances do not decay. The experimental data on the oscillation amplitude and the decay rate are in excellent agreement with calculations based on the SW dispersion [solid curves in Fig. 2(b); Ref. [30]].

\section{CONCLUSIONS}

Summarizing the experimental data and their analysis, we have demonstrated that a train of optical pump pulses can drive the magnetization within the illuminated area, thereby exciting magnons. The magnons become spread by propagation from the excitation spot, and their superposition is observable as SWs. Therefore, the excited area can be considered as a coherent magnon "cloud," which serves as a source of SWs with parameters controllable by the details of the experimental protocol such as the laser illumination or the magnetic field.

The magnon cloud has several outstanding properties. In particular, the amplitude of the oscillations in this cloud can be significantly increased if the magnetization oscillation frequency is tuned by the magnetic field of moderate strength such that it becomes synchronized with the laser pulses. The enhancement factor can be as large as 9.3 for a magnetic mode of high quality factor $(Q \sim 232)$. Such enhancement has allowed us to observe a magnetization mode that is generally obscured in an experiment using single-pulse excitation by other modes of lower quality factor. Our technique therefore can be applied as a highly sensitive spectroscopy tool for resolving magnetization precession modes. Remarkably, the decay rate of the precession amplitude strongly depends on the distance from the optical pump. At some distance, the rate becomes so small that the oscillation hardly undergoes a decay anymore. Moreover, the periodic pumping additionally brings two important features for SW propagation: It significantly increases their propagation distance, and it also provides the possibility to tune the directionality of propagation.

Generally, optical excitation of magnetic oscillations represents a detailed control tool of the magnetization distribution at subterahertz time scales. In combination with focusing by plasmonic antennas, the spatial distribution can also be tailored on subwavelength scales, which is of prime importance for quantum information processing based on SWs [4]. The tunable, quasistationary magnon cloud as a spin-wave source might significantly broaden the functionality of this approach. The next step forward will be implementation of plasmonic structures to further enhance the magnetization precession by concentrating the optical fields in a nanometer-thick magnetic film with a spot size of 
less than $100 \mathrm{~nm}$ in diameter [25]. Indeed, while a huge plasmonics-mediated increase of the direct magneto-optical effects was demonstrated recently [36], the plasmonic boost of the inverse magneto-optical effects is still waiting for its practical implementation [15].

\section{ACKNOWLEDGEMENTS}

M. J., I. A. A., D. R. Y., and M. B. acknowledge financial support by the Deutsche Forschungsgemeinschaft (Project No. AK40/7-1 and the International Collaborative Research Centre 160), while V.I.B. and A.K.Z. acknowledge financial support by the Russian President Grant (Project No. MD-1615.2017.2). Russian Foundation for Basic Research (Project No. 16-52-45061).

[1] S. O. Demokritov and A. N. Slavin, Magnonics: From Fundamentals to Applications (Springer, Heidelberg, 2013).

[2] A. V.Chumak, V. I. Vasyuchka, A. A. Serga, and B. Hillebrands, Magnon Spintronics, Nat. Phys. 11, 453 (2015).

[3] B. Lenk, H. Ulrichs, F. Garbs, and M. Münzenberg, The Building Blocks of Magnonics, Phys. Rep. 507, 107 (2011).

[4] Y. Tabuchi, S. Ishino, A. Noguchi, T. Ishikawa, R. Yamazaki, K. Usami, and Y. Nakamura, Coherent Coupling between a Ferromagnetic Magnon and a Superconducting Qubit, Science 349, 405 (2015).

[5] J. H. Wesenberg, A. Ardavan, G. A. D. Briggs, J. J. L. Morton, R. J. Schoelkopf, D. I. Schuster, and K. Mølmer Quantum Computing with an Electron Spin Ensemble, Phys. Rev. Lett. 103, 070502 (2009).

[6] V. E. Demidov, M. P. Kostylev, K. Rott, P. Krzysteczko, G. Reiss, and Sergej O. Demokritov, Excitation of Microwaveguide Modes by a Stripe Antenna, Appl. Phys. Lett. 95, 112509 (2009).

[7] X. Zhang, C. Zou, N. Zhu, F. Marquardt, L. Jiang, and Hong X. Tang, Magnon Dark Modes and Gradient Memory, Nat. Commun. 6, 8914 (2015).

[8] T. Satoh, Y. Terui, R. Moriya, B. A. Ivanov, K. Ando, E. Saitoh, T. Shimura, and K. Kuroda, Directional Control of Spin-Wave Emission by Spatially Shaped Light, Nat. Photonics 6, 662 (2012).

[9] I. Yoshimine, T. Satoh, R. Iida, A. Stupakiewicz, A. Maziewski, and T. Shimura, Phase-Controllable Spin Wave Generation in Iron Garnet by Linearly Polarized Light Pulses, J. Appl. Phys. 116, 043907 (2014).

[10] S. Parchenko, A. Stupakiewicz, I. Yoshimine, T. Satoh, and A. Maziewski, Wide Frequencies Range of Spin Excitations in a Rare-Earth Bi-Doped Iron Garnet with a Giant Faraday Rotation, Appl. Phys. Lett. 103, 172402 (2013).

[11] M. van Kampen, C. Jozsa, J. T. Kohlhepp, P. LeClair, L. Lagae, W. J. M. de Jonge, and B. Koopmans, All-Optical Probe of Coherent Spin Waves, Phys. Rev. Lett. 88, 227201 (2002).

[12] Y. Au, M. Dvornik, T. Davison, E. Ahmad, P. S. Keatley, A. Vansteenkiste, B. Van Waeyenberge, and V. V. Kruglyak, Direct Excitation of Propagating Spin Waves by Focused
Ultrashort Optical Pulses, Phys. Rev. Lett. 110, 097201 (2013).

[13] B. Lenk, G. Eilers, J. Hamrle, and M. Münzenberg, SpinWave Population in Nickel after Femtosecond Laser Pulse Excitation, Phys. Rev. B 82, 134443 (2010).

[14] E. Beaurepaire, J. Merle, A. Daunois, and J. Bigot, Ultrafast Spin Dynamics in Ferromagnetic Nickel, Phys. Rev. Lett. 76, 4250 (1996).

[15] V.I. Belotelov and A. K. Zvezdin, Inverse Transverse Magneto-Optical Kerr Effect, Phys. Rev. B 86, 155133 (2012).

[16] J. Bigot, M. Vomir, and E. Beaurepaire, Coherent Ultrafast Magnetism Induced by Femtosecond Laser Pulses, Nat. Phys. 5, 515 (2009).

[17] B. Koene, M. Deb, E. Popova, N. Keller, Th. Rasing, and A. Kirilyuk, Excitation of Magnetic Precession in Bismuth Iron Garnet via a Polarization-Independent Impulsive Photomagnetic Effect, Phys. Rev. B 91, 184415 (2015).

[18] F. Atoneche, A. M. Kalashnikova, A. V. Kimel, A. Stupakiewicz, A. Maziewski, A. Kirilyuk, and Th. Rasing, Large Ultrafast Photoinduced Magnetic Anisotropy in a Cobalt-Substituted Yttrium Iron Garnet, Phys. Rev. B 81, 214440 (2010).

[19] A. V. Kimel, A. Kirilyuk, P. A. Usachev, R. V. Pisarev, A. M. Balbashov, and Th. Rasing Ultrafast Non-thermal Control of Magnetization by Instantaneous Photomagnetic Pulses, Nature (London) 435, 655 (2005).

[20] F. Hansteen, A. V. Kimel, A. Kirilyuk, and Th. Rasing, Nonthermal Ultrafast Optical Control of the Magnetization in Garnet Films, Phys. Rev. B 73, 014421 (2006).

[21] C. D. Stanciu, F. Hansteen, A. V. Kimel, A. Kirilyuk, A. Tsukamoto, A. Itoh, and Th. Rasing All-Optical Magnetic Recording with Circularly Polarized Light, Phys. Rev. Lett. 99, 047601 (2007).

[22] A. H. M. Reid, A. V. Kimel, A. Kirilyuk, J. F. Gregg, and Th. Rasing, Optical Excitation of a Forbidden Magnetic Resonance Mode in a Doped Lutetium-Iron-Garnet Film via the Inverse Faraday Effect, Phys. Rev. Lett. 105, 107402 (2010).

[23] M. Deb, M. Vomir, J. Rehspringer, and J. Bigot, Ultrafast Optical Control of Magnetization Dynamics in Polycrystalline Bismuth Doped Iron Garnet Thin Films, Appl. Phys. Lett. 107, 252404 (2015).

[24] D. Bossini, V. I. Belotelov, A. K. Zvezdin, A. N. Kalish, and A. V. Kimel, Magnetoplasmonics and Femtosecond OptoMagnetism at the Nanoscale, ACS Photonics 3, 1385 (2016).

[25] T. Liu et al. Nanoscale Confinement of All-Optical Magnetic Switching in TbFeCo-Competition with Nanoscale Heterogeneity, Nano Lett. 15, 6862 (2015).

[26] K. Uchida, H. Adachi, D. Kikuchi, S. Ito, Z. Qiu, S. Maekawa, and E. Saitoh Generation of Spin Currents by Surface Plasmon Resonance, Nat. Commun. 6, 5910 (2015).

[27] L. P. Pitaevskii, Electric Forces in a Transparent Dispersive Medium, J. Exp. Theor. Phys. 39, 1450 (1960).

[28] D. Bossini, S. Dal Conte, Y. Hashimoto, A. Secchi, R. V. Pisarev, Th. Rasing, G. Cerullo, and A. V. Kimel, Macrospin Dynamics in Antiferromagnets Triggered by Sub-20 Femtosecond Injection of Nanomagnons, Nat. Commun. 7, 10645 (2016). 
[29] J. Kim, M. Vomir, and J. Bigot, Controlling the Spins Angular Momentum in Ferromagnets with Sequences of Picosecond Acoustic Pulses, Sci. Rep. 5, 8511 (2015).

[30] See Supplemental Material at http://link.aps.org/ supplemental/10.1103/PhysRevX.7.021009 for relation between the observed Faraday rotation angle and the polar angle of the magnetization precession, derivations of Eqs. (2) and (3), fitting parameters, and numerical calculation of spin waves propagation.

[31] R. Gebs, G. Klatt, C. Janke, T. Dekorsy, and A. Bartels, High-Speed Asynchronous Optical Sampling with Sub-50 fs Time Resolution, Opt. Express 18, 5974 (2010).

[32] N. Krauß, G. Schafer, J. Flock, O. Kliebisch, C. Li, H. G. Barros, D. C. Heinecke, and T. Dekorsy, Two-Colour High-Speed Asynchronous Optical Sampling Based on
Offset-Stabilized $\mathrm{Yb}: \mathrm{KYW}$ and Ti:sapphire Oscillators, Opt. Express 23, 18288 (2015).

[33] A. Kirilyuk, A. V. Kimel, and T. Rasing, Ultrafast Optical Manipulation of Magnetic Order, Rev. Mod. Phys. 82, 2731 (2010).

[34] D. Bossini and T. Rasing, Femtosecond Optomagnetism in Dielectric Antiferromagnets, Phys. Scr. 92, 024002 (2017).

[35] R. Gieniusz and L. Smoczyński, Magnetostatic Spin Waves in (111)-Oriented Thin Garnet Films with Combined Cubic and Uniaxial Anisotropies, J. Magn. Magn. Mater. 66, 366 (1987).

[36] V. I. Belotelov, I. A. Akimov, M. Pohl, V. A. Kotov, S. Kasture, A. S. Vengurlekar, A. Venu Gopal, D. R. Yakovlev, A. K. Zvezdin, and M. Bayer, Enhanced Magneto-Optical Effects in Magnetoplasmonic Crystals, Nat. Nanotechnol. 6, 370 (2011). 